\title{
Há irracionalidades no consumo de inibidores de apetite no Brasil? Uma análise farmacoeconométrica de dados em painel
}

\author{
Are there irrationalities in the consumption of anti-obesity drugs \\ in Brazil? A pharmaco-econometric analysis of panel datasets
}

Daniel Marques Mota ${ }^{1}$

Márcia Gonçalves de Oliveira ${ }^{2}$

Rafael Filiacci Bovi ${ }^{2}$

Sidarta Figueredo Silva ${ }^{2}$

Jeane Araújo Fernandes Cunha ${ }^{2}$

José Angelo Divino ${ }^{3}$

\footnotetext{
${ }^{1}$ Programa de PósGraduação em Epidemiologia, Faculdade de Medicina, Universidade Federal do Rio Grande do Sul. R. Ramiro Barcelos 2400/2 ${ }^{\circ}$ andar/414, Santa Cecília. 90.035-003 Porto Alegre RS Brasil. dmarques2003@yahoo.com.br ${ }^{2}$ Coordenação do Sistema Nacional de Gerenciamento de Produtos Controlados, Agência Nacional de Vigilância Sanitária. ${ }^{3}$ Diretoria do Mestrado e Doutorado em Economia, Universidade Católica de Brasília.
}

\begin{abstract}
The scope of this study is to analyze the determinants of the use of appetite suppressants (amfepramone, femproporex, mazindol and sibutramine) through the estimation of a dynamic panel dataset model for the Brazilian state capitals and the Federal District (DF) in the period from 2009 to 2011. The results show that consumption of appetite suppressants did not follow the geographic distribution of overweight and obese individuals across the capitals and DF. There is a recurrent consumption of appetite inhibitors, in which $79 \%$ of the current consumption of these drugs is explained by past consumption. Among the variables that explain the use of inhibitors, the percentage of obese adults, the percentage of adults who habitually consume fruit and vegetables, and the coverage rate of health plans stand out. The pharmaco-econometric analysis suggests that there are problems in the rational use of appetite suppressants in the Brazilian state capitals and the Federal District with respect to both the combined consumption of these drugs with other medicines - deemed illegal by the Federal Council of Medicine and ANVISA and in the therapeutic prescription of these products.
\end{abstract}

Key words Regression analysis, Appetite suppressants, Pharmaceutical economics, Obesity, Rational drug use
Resumo Este estudo busca analisar os determinantes do consumo de inibidores de apetite (anfepramona, femproporex, mazindol e sibutramina) por meio da estimação de um modelo dinâmico de dados em painel para as capitais brasileiras e do Distrito Federal (DF) no período de 2009 a 2011. Os resultados revelam que o consumo de inibidores de apetite não acompanhou a distribuição geográfica dos indivíduos com excesso de peso e com obesidade nas unidades estudadas. Do consumo recorrente de inibidores, 79\% são explicados pelo ocorrido no passado. Dentre as variáveis que explicam o consumo de inibidores, destacam-se os percentuais de adultos com obesidade e que dos que consomem frutas e hortaliças e a taxa de cobertura de planos de saúde. A análise farmacoeconométrica sugere que há problemas no uso racional dos inibidores de apetite nas capitais brasileiras e no DF, seja no que tange ao consumo desses medicamentos com outros fármacos - considerados ilegais pelo Conselho Federal de Medicina e pela Anvisa - e, também, na indicação terapêutica de uso desses produtos.

Palavras-chave Análise de regressão, Depressores do apetite, Economia farmacêutica, Obesidade, Uso racional de medicamentos 


\section{Introdução}

O Brasil foi mencionado, por muitos anos, como um dos maiores consumidores de inibidores de apetite do mundo com evidências de problemas na prescrição, venda e uso desses medicamentos $^{1-9}$. À época, inibidores de apetite vendidos no país, como anfepramona, femproporex e mazindol ${ }^{10}$, não estavam sendo mais comercializados em vários países, pois a relação benefíciorisco é desfavorável ${ }^{11-13}$. Em 2011, a Agência Nacional de Vigilância Sanitária (Anvisa) determinou o cancelamento dos registros dos medicamentos anfepramona, femproporex e mazindol e fixou regras mais rígidas de controle sanitário sobre a venda de sibutramina no país ${ }^{14}$. Essas medidas, aliadas a outras intervenções não necessariamente regulatórias, podem ter contribuído para o controle e redução do uso irracional desses produtos.

O uso irracional é a utilização de medicamentos não condizente com a definição de uso racional estabelecida pela Organização Mundial da Saúde (OMS), em 1985, a saber: "Os pacientes recebem a medicamento adequado às suas necessidades clínicas, nas doses correspondentes às suas características individuais, durante um período de tempo adequado e ao menor custo possível para eles e para a comunidade"15. Essa prática tem se tornado um dos graves problemas de saúde pública, ocasionando danos consideráveis aos pacientes, como baixos resultados terapêuticos, empobrecimento do indivíduo (despesas grandes e desnecessárias), surgimento de reações adversas e erros de medicação e aumento da morbidade e óbito. Outro aspecto é que o uso irracional de medicamentos também sobrecarrega negativamente, em termos econômicos e assistenciais, os sistemas públicos de saúde de muitos países ${ }^{16}$.

Vários são os fatores que podem contribuir para o uso irracional de medicamentos, como: i) carência de conhecimento médico, especialmente para aqueles insuficientemente qualificados; ii) hábitos de prescrição não baseados em evidências científicas; iii) fatores sociais e culturais que contribuem para a indução da demanda por medicamentos; iv) fatores econômicos, pois torna-se motivo de lucro para diferentes atores da cadeia de utilização do medicamento; e v) fatores informativos, incluindo a pouca disponibilidade de informações imparciais destinadas aos prescritores que possam contrapor a informação excessiva e, muitas vezes, tendenciosa dos laboratórios farmacêuticos ${ }^{16,17}$. Este estudo tem por objetivo examinar a relação entre o consumo de medicamentos inibidores de apetite e as variáveis socioeconômicas, assistências, de condição fisiológica e de hábitos de vida saudável nas capitais brasileiras e no Distrito Federal (DF).

\section{Métodos}

Trata-se de um estudo farmacoeconométrico ${ }^{7,18}$ em que foi utilizado um modelo de regressão linear dinâmico de dados em painel das capitais brasileiras e do Distrito Federal nos anos de 2009 a 2011. Nessa modelagem, a variável dependente defasada no tempo é incluída entre as explicativas e os dados são observados para distintas capitais brasileiras e o Distrito Federal ao longo do tempo.

A disponibilidade de dados de corte para as variáveis selecionadas e para o período de tempo estudado foi o principal motivo para a escolha das unidades de análise (capitais brasileiras e DF). A justificativa do uso do modelo de regressão linear dinâmico se deve à intenção de verificar se o consumo recente de inibidores de apetite é influenciado pelo uso anterior desses produtos. A escolha pela técnica de dados em painel deve-se a que, no período analisado, ocorreram mudanças, em termos regulatórios, que podem ter influenciado o consumo desses medicamentos no conjunto das unidades analisadas ${ }^{14,19}$. Esse modelo considera essas mudanças, pois utiliza informação temporal e unitária que impõe maior controle aos efeitos não observáveis ou fatores de confundimento, bem como tem uma capacidade de controlar ainda a heterogeneidade existente entre as capitais e o DF. Uma discussão detalhada sobre essa modelagem poder ser obtida, por exemplo, em $\mathrm{Hsiao}^{20}$.

O Quadro 1 apresenta as variáveis explicativas selecionadas para o modelo de regressão linear e as fontes de obtenção dos dados. Essas variáveis foram apontadas na literatura técnicocientífica ${ }^{4,7,11}$ ou consideradas pelos autores como tendo relação com o consumo de inibidores de apetite, representado pelo conjunto dos medicamentos anfepramona, femproporex, mazindol e sibutramina (CIA). Esses medicamentos, no período avaliado, faziam parte das listas da Portaria SVS/MS no 344, de 12 de maio de $1998^{21}$. Os medicamentos que contêm fluoxetina ou clordiazepóxido pertencem à lista $\mathrm{C}_{1}$ enquanto que os inibidores de apetite estão na lista $\mathrm{B}_{2}$, a qual pressupõe um maior controle sanitário.

A variável explicativa percentual de pessoas com excesso de peso (EXP) foi utilizada como 
Quadro 1. Variáveis utilizadas no modelo de regressão linear dinâmico com dados em painel.

\begin{tabular}{|c|c|c|c|}
\hline Sigla & Nome (tipo de variável) & Descrição & Fonte \\
\hline CIA & $\begin{array}{l}\text { Consumo de inibidores de } \\
\text { apetite (variável } \\
\text { dependente) }\end{array}$ & $\begin{array}{l}\text { Consumo em mg/per capita* de inibidores de } \\
\text { apetite em formulações industrializadas e } \\
\text { manipuladas nas capitais brasileiras e no DF }\end{array}$ & $\mathrm{SNGPC}^{* *}$ \\
\hline CFF & $\begin{array}{l}\text { Consumo de fluoxetina } \\
\text { (variável explicativa) }\end{array}$ & $\begin{array}{l}\text { Consumo em mg/per capita* de fluoxetina em } \\
\text { formulações industrializadas e manipuladas nas } \\
\text { capitais brasileiras e no DF }\end{array}$ & $\mathrm{SNGPC}^{* *}$ \\
\hline СCP & $\begin{array}{l}\text { Consumo de } \\
\text { clordiazepóxido (variável } \\
\text { explicativa) }\end{array}$ & $\begin{array}{l}\text { Consumo em mg/per capita* de } \\
\text { clordiazepóxido em formulações } \\
\text { industrializadas e manipuladas nas capitais } \\
\text { brasileiras e no DF }\end{array}$ & $\mathrm{SNGPC}^{* *}$ \\
\hline FEM & $\begin{array}{l}\text { Percentual de mulheres } \\
\text { (variável explicativa) }\end{array}$ & $\begin{array}{l}\text { Percentual de mulheres com idade entre } 18 \text { e } \\
64 \text { anos nas capitais brasileiras e no DF }\end{array}$ & DATASUS ${ }^{22}$ \\
\hline OBE & $\begin{array}{l}\text { Percentual de adultos com } \\
\text { obesidade (variável } \\
\text { explicativa) }\end{array}$ & $\begin{array}{l}\text { Percentual de adultos ( }>18 \text { anos) com } \\
\text { obesidade (Índice de Massa Corporal }>30 \mathrm{~kg} / \\
\mathrm{m}^{2} \text { ) nas capitais brasileiras e no DF }\end{array}$ & $\begin{array}{l}\text { VIGITEL/ } \\
\text { Ministério da } \\
\text { Saúde }^{23}\end{array}$ \\
\hline EXP & $\begin{array}{c}\text { Percentual de adultos com } \\
\text { excesso de peso (variável } \\
\text { explicativa) }\end{array}$ & $\begin{array}{l}\text { Percentual de adultos ( }>18 \text { anos) com excesso } \\
\text { de peso (Índice de Massa Corporal }>25 \mathrm{~kg} / \mathrm{m}^{2} \text { ) } \\
\text { nas capitais brasileiras e no DF }\end{array}$ & $\begin{array}{l}\text { VIGITEL/ } \\
\text { Ministério da } \\
\text { Saúde }^{23}\end{array}$ \\
\hline PFH & $\begin{array}{l}\text { Percentual de adultos que } \\
\text { consomem frutas e } \\
\text { hortaliças (variável } \\
\text { explicativa) }\end{array}$ & $\begin{array}{l}\text { Percentual de adultos ( }>18 \text { anos) que } \\
\text { consomem cinco ou mais porções diárias de } \\
\text { frutas e hortaliças nas capitais brasileiras e no } \\
\text { DF }\end{array}$ & $\begin{array}{l}\text { VIGITEL/ } \\
\text { Ministério da } \\
\text { Saúde }^{23}\end{array}$ \\
\hline PLS & $\begin{array}{l}\text { Taxa, em percentual, de } \\
\text { cobertura de planos de } \\
\text { saúde (variável explicativa) }\end{array}$ & $\begin{array}{l}\text { Taxa, em percentual, de cobertura de planos de } \\
\text { saúde de beneficiários na faixa etária de } 15 \text { a } 69 \\
\text { anos nas capitais brasileiras e no DF }\end{array}$ & $\begin{array}{l}\text { Agência } \\
\text { Nacional de } \\
\text { Saúde } \\
\text { Suplementar } \\
\text { Su }^{24}\end{array}$ \\
\hline
\end{tabular}

"Ajustado para a população de 18 a 64 anos de idade. ** O SNGPC é uma sistema de informação de vigilância sanitária, gerenciado pela Anvisa, que coleta dados de compra e venda dos medicamentos da Portaria SVS/MS n ${ }^{\circ} 344 / 1998^{21}$ comercializados em farmácias e drogarias privadas do país. Esses dados foram utilizados como proxy do consumo dos medicamentos citados no quadro acima nas capitais brasileiras e no DF. Obs.: i. A variável FEM, para o ano de 2011, foi estimada a partir da diferença entre os valores de 2010 e 2009. Essa diferença foi acrescentada ao valor de 2010, obtendo a quantidade estimada para 2011; ii. Os medicamentos anfepramona, femproporex e mazindol tiveram a comercialização cancelada no país a partir do dia 9 de dezembro de 2011; e iii. O uso da faixa etária para a variável PLS foi condicionado à disponibilidade do dado pela fonte consulta.

proxy para excesso de peso com comorbidade enquanto que a variável percentual de pessoas que ingerem porções de frutas e hortaliças (PFH) foi utilizada como proxy de indivíduos com hábitos de vida mais saudável.

Os dados com informações agregadas por capitais brasileiras e DF foram obtidos do Sistema Nacional de Gerenciamento de Produtos Controlados (SNGPC) da Anvisa (consumo per capita de medicamentos), do inquérito telefônico Vigitel sobre fatores de risco e proteção para doenças crônicas não transmissíveis do Ministé- rio da Saúde (percentual de adultos com excesso de peso, obesidade e que consomem frutas e hortaliças) e da Agência Nacional de Saúde Suplementar (taxa, em percentual, de cobertura de planos de saúde). Os dados do percentual de mulheres com idade entre 18 e 64 anos foram extraídos do sítio eletrônico do Departamento de Informática do SUS do Ministério da Saúde (Datasus) (Quadro 1).

A representação formal do modelo de regressão linear dinâmico com dados em painel está apresentada a seguir: 


$$
\ln Y_{i t}=\alpha+\rho \ln Y_{i t-1}+\beta \ln X_{i t}+\delta_{i}+\varepsilon_{i t}(1)
$$

Onde:

$\mathrm{i}=1,2, \ldots, 27$ representa as capitais brasileiras e o Distrito Federal;

$\mathrm{t}=2009$, 2010 e 2011 ;

$\alpha=$ intercepto ou constante da regressão;

$\rho=$ coeficiente da variável dependente defasada;

$\beta=$ vetor de coeficientes das variáveis explicativas;

$\ln Y_{i t}=$ consumo de inibidores de apetite nas capitais e no DF em logaritmo natural;

$\ln Y_{i{ }^{\prime} 1}=$ variável dependente defasada em logaritmo natural;

$\ln X_{i t}=$ conjunto das variáveis explicativas em logaritmo natural;

$\delta_{\mathrm{i}}=$ heterogeneidade individual específica; e,

$\varepsilon_{i t}=$ termo de erro aleatório, variante no tempo e entre as unidades analisadas.

A estimação consistente e eficiente dos parâmetros foi obtida a partir do Método dos Momentos Generalizado, versão dois passos (twosteps). Esse método é uma das técnicas mais avançadas em farmacoeconometria, pois ajuda a evitar problemas de endogeneidade e causalidade reversa. Com a intenção de estabilizar a variância das séries e tornar a interpretação dos coeficientes estimados na forma de elasticidades, optou-se pela transformação logarítmica (ln) das variáveis estudadas. Os coeficientes estimados para cada variável no modelo de regressão duplo-log indicam variações percentuais no consumo de inibidores de apetite, de acordo com mudanças percentuais na variável analisada. Por exemplo, se o coeficiente para $\ln C F F$ for 0,40 , indica que a variação positiva no consumo de fluoxetina - de $1 \%$ - ocasiona uma elevação média de $0,40 \%$ no consumo de inibidores de apetite. A robustez do modelo foi avaliada com o Teste de Sargan e o Teste de Wald. O software Gretl, versão 1.9.8., foi utilizado para a estimação do modelo.

Os resultados analisados a seguir correspondem à estimação da equação (1), modelo que visa identificar o tipo de relação existente entre o consumo de inibidores de apetite e as variáveis explicativas. Antes, é apresentada uma caracterização sanitária dos medicamentos estudados e uma análise descritiva do consumo dos inibidores de apetite, de fluoxetina e de clordiazepóxido nas capitais brasileiras e no Distrito Federal, bem como do percentual de indivíduos com excesso de peso e com obesidade. Os cálculos das estimativas de variação percentual $(\Delta)$ de aumento ou redução no consumo dos medicamentos 2011 e 2009 foram feitos a partir da subtração da va- riação percentual desses produtos pela variação percentual dos arquivos de movimentação de compra e venda dos medicamentos enviados e aceitos pelo SNGPC relacionados com cada capital e DF. A variação percentual foi também calculada para os adultos com excesso de peso e com obesidade. Os dados de consumo dos medicamentos foram gerados no SNGPC para a este estudo na $1^{\text {a }}$ quinzena de abril de 2012 .

Os dados utilizados são públicos e disponíveis em sítios eletrônicos ou foram obtidos por meio de contato institucional. Ressalta-se ainda que pessoas não foram incluídas como sujeitos da pesquisa neste estudo e a identificação das capitais e DF não as colocam em situação de constrangimento. Neste sentido, os autores entenderam que não foi necessário o envio deste estudo para apreciação de algum Comitê de Ética em Pesquisa.

\section{Resultados}

Entre 2009 e 2011, houve um descompasso em relação ao consumo de inibidores de apetite nas capitais brasileiras e no DF e as pessoas com excesso de peso e obesidade (Tabela 1). Em todas as capitais e DF ocorreu uma redução no consumo de inibidores de apetite, à exceção de Vitória, na qual se registrou um aumento real de $82,32 \%$. Em relação à variação percentual de pessoas com excesso de peso, em oito capitais ocorreu uma diminuição nessa variável, enquanto que para os indivíduos obesos isso foi verificado em quatro unidades analisadas, a saber: Aracaju (-10,98\%), Porto Velho $(-6,82 \%)$, Rio de Janeiro $(-6,78 \%)$ e Salvador $(-1,97 \%)$. As variações percentuais no consumo de fluoxetina e clordiazepóxido, de maneira geral, acompanharam o perfil registrado para os inibidores de apetite. Em 19 unidades analisadas ocorreu uma redução no consumo de fluoxetina, enquanto que para o clordiazepóxido esse número chegou a 25 nas capitais e no DF. Destaca-se que Vitória foi a única capital brasileira que apresentou um incremento percentual em todas as variáveis estudadas (Tabela 1).

As Figuras 1 e 2 apresentam a distribuição de indivíduos com excesso de peso e com obesidade em percentuais e do consumo de inibidores de apetite (CIA) em mg/per capita para algumas capitais brasileiras e DF, agrupadas por regiões do país.

Na região Centro-Oeste, Campo Grande (2009 e 2010) e Cuiabá (2011) registraram os maiores percentuais de indivíduos com excesso 
Tabela 1. Variação percentual $(\Delta)$ do consumo de inibidores de apetite (CIA), de fluoxetina (CFF) e de clordiazepóxido (CCP), adultos com excesso de peso (EXP) e obesos (OBE) entre 2011 e 2009, segundo capitais brasileiras e DF, agrupadas por regiões do país.

\begin{tabular}{|c|c|c|c|c|c|}
\hline UF & $\Delta_{2011-2009}$ CIA & $\Delta_{2011-2009} \mathrm{CFF}$ & $\Delta_{2011-2009} \mathrm{CCP}$ & $\Delta_{2011-2009}$ EXP & $\Delta_{2011-2009} \mathrm{OBE}$ \\
\hline \multicolumn{6}{|l|}{ Região Norte } \\
\hline Belém & $-129,82$ & $-52,38$ & $-64,67$ & 3,39 & 3,12 \\
\hline Boa Vista & $-105,75$ & $-59,70$ & $-37,13$ & $-1,02$ & 2,36 \\
\hline Macapá & $-73,93$ & 77,76 & $-161,52$ & 17,70 & 41,72 \\
\hline Manaus & $-101,66$ & $-66,13$ & $-45,46$ & 13,60 & 18,67 \\
\hline Palmas & $-157,47$ & $-141,22$ & -190.17 & 6,90 & 42,05 \\
\hline Porto Velho & $-228,93$ & $-230,57$ & $-250,50$ & 0,82 & $-6,82$ \\
\hline Rio Branco & $-101,06$ & 0,85 & $-71,06$ & $-7,85$ & 0,0 \\
\hline \multicolumn{6}{|l|}{ Região Nordeste } \\
\hline Aracaju & $-87,99$ & 10,96 & $-58,28$ & $-6,12$ & $-10,98$ \\
\hline Fortaleza & $-147,58$ & 76,96 & $-47,59$ & 14,26 & 20,26 \\
\hline João Pessoa & $-86,30$ & $-46,28$ & $-44,99$ & 16,08 & 15,45 \\
\hline Maceió & $-62,87$ & 0,81 & $-74,58$ & 27,95 & 36,64 \\
\hline Natal & $-77,75$ & $-15,90$ & $-28,12$ & 14,95 & 39,10 \\
\hline Recife & $-75,38$ & 3,17 & 25,35 & 3,29 & 7,25 \\
\hline Salvador & $-181,50$ & $-75,53$ & $-177,13$ & $-1,10$ & $-1,97$ \\
\hline São Luís & $-50,89$ & $-6,06$ & $-34,30$ & $-1,24$ & 6,61 \\
\hline Teresina & $-157,07$ & $-69,31$ & $-191,42$ & 12,94 & 5,79 \\
\hline \multicolumn{6}{|c|}{ Região Centro-Oeste } \\
\hline Campo Grande & $-277,16$ & $-250,89$ & $-208,74$ & $-2,95$ & 4,62 \\
\hline Cuiabá & $-23,69$ & 23,02 & $-38,13$ & 10,71 & 23,74 \\
\hline DF & $-59,85$ & $-23,16$ & $-57,93$ & 35,64 & 61,29 \\
\hline Goiânia & $-39,46$ & $-27,97$ & $-62,60$ & 2,62 & 16,67 \\
\hline \multicolumn{6}{|l|}{ Região Sudeste } \\
\hline Belo Horizonte & $-26,37$ & $-26,45$ & $-36,13$ & 13,53 & 26,79 \\
\hline Rio de Janeiro & $-54,26$ & $-7,16$ & $-63,41$ & $-1,59$ & $-6,78$ \\
\hline São Paulo & $-111,61$ & $-81,53$ & $-115,39$ & $-5,15$ & 18,32 \\
\hline Vitória & 82,32 & 109,44 & 202,40 & 2,16 & 12,98 \\
\hline \multicolumn{6}{|l|}{ Região Sul } \\
\hline Curitiba & $-19,40$ & $-0,24$ & $-58,17$ & 9,89 & 25,58 \\
\hline Florianópolis & $-88,20$ & $-28,56$ & $-76,45$ & 7,11 & 17,32 \\
\hline Porto Alegre & $-97,63$ & $-69,32$ & $-120,47$ & 20,17 & 37,06 \\
\hline
\end{tabular}

de peso. Os menores valores foram contabilizados no DF (2009) e Goiânia (2010 e 2011). No caso de indivíduos obesos, Campo Grande (2009 e 2011) e Cuiabá (2010) obtiveram os maiores percentuais. A capital federal (2009 e 2010) e Goiânia (2011) registraram os menores valores para essa condição fisiológica. No entanto, no triênio estudado, o maior valor do consumo de inibidores de apetite foi registrado em Goiânia e o menor em Campo Grande (Figura 1).

Na região Sudeste, a capital fluminense obteve o maior número de indivíduos com excesso de peso e com obesidade e Belo Horizonte registrou os menores valores para essas condições fisiológicas. Porém, para o consumo de inibidores de apetite, Vitória e Belo Horizonte contabilizaram os maiores valores (Figura 2). Na região Sul, Porto Alegre registrou o maior percentual de indivíduos com excesso de peso. Em relação à obesidade, Porto Alegre (2009 e 2011) e Curitiba (2010) registraram o maior número de indivíduos nessa condição. A capital do estado de Santa Catarina contabilizou o menor valor percentual para ambas as condições fisiológicas analisadas. O consumo de inibidores de apetite não seguiu a distribuição geográfica para os indivíduos com excesso de peso e com obesidade (Figura 2). O menor consumo desse grupo de medicamentos foi registrado para a capital gaúcha, enquanto que Florianópolis (2009) e Curitiba 


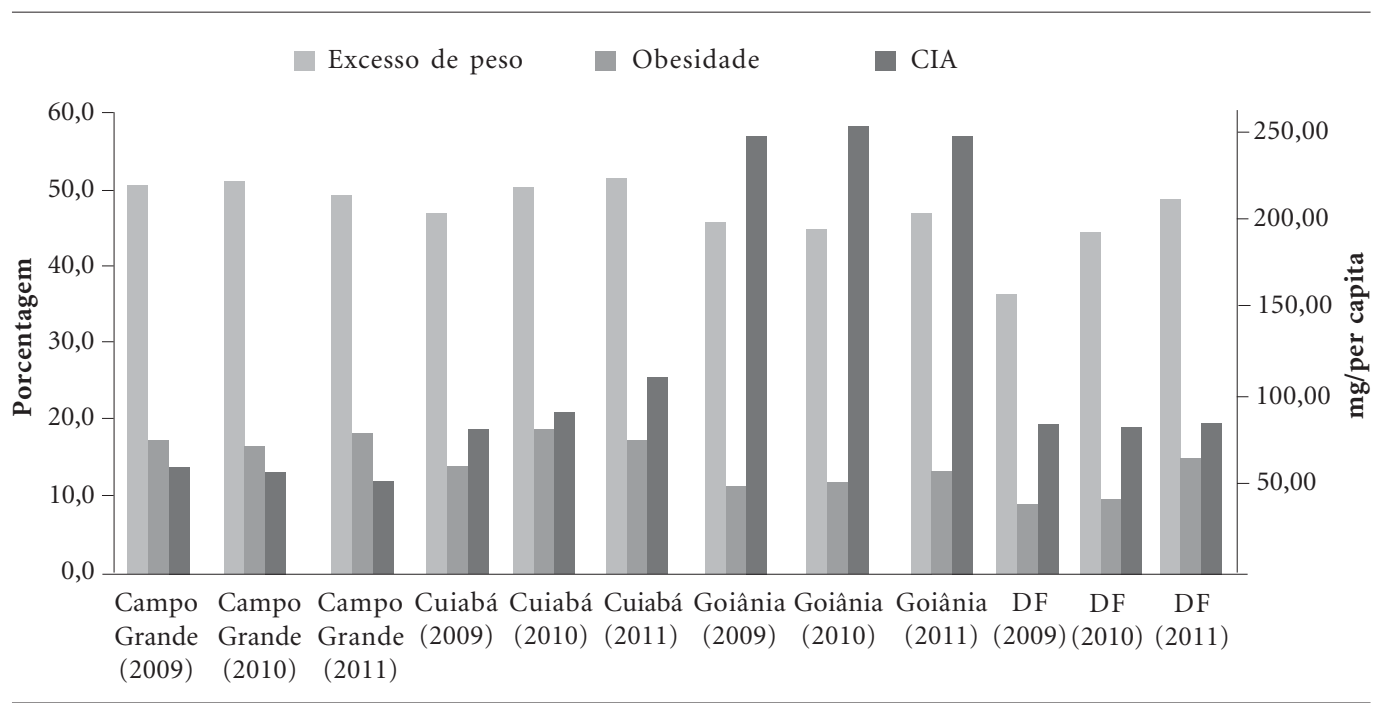

Figura 1. Distribuição de indivíduos com excesso de peso e com obesidade e do consumo de inibidores de apetite (CIA) nas capitais e DF da região Centro-Oeste, 2009-2011.

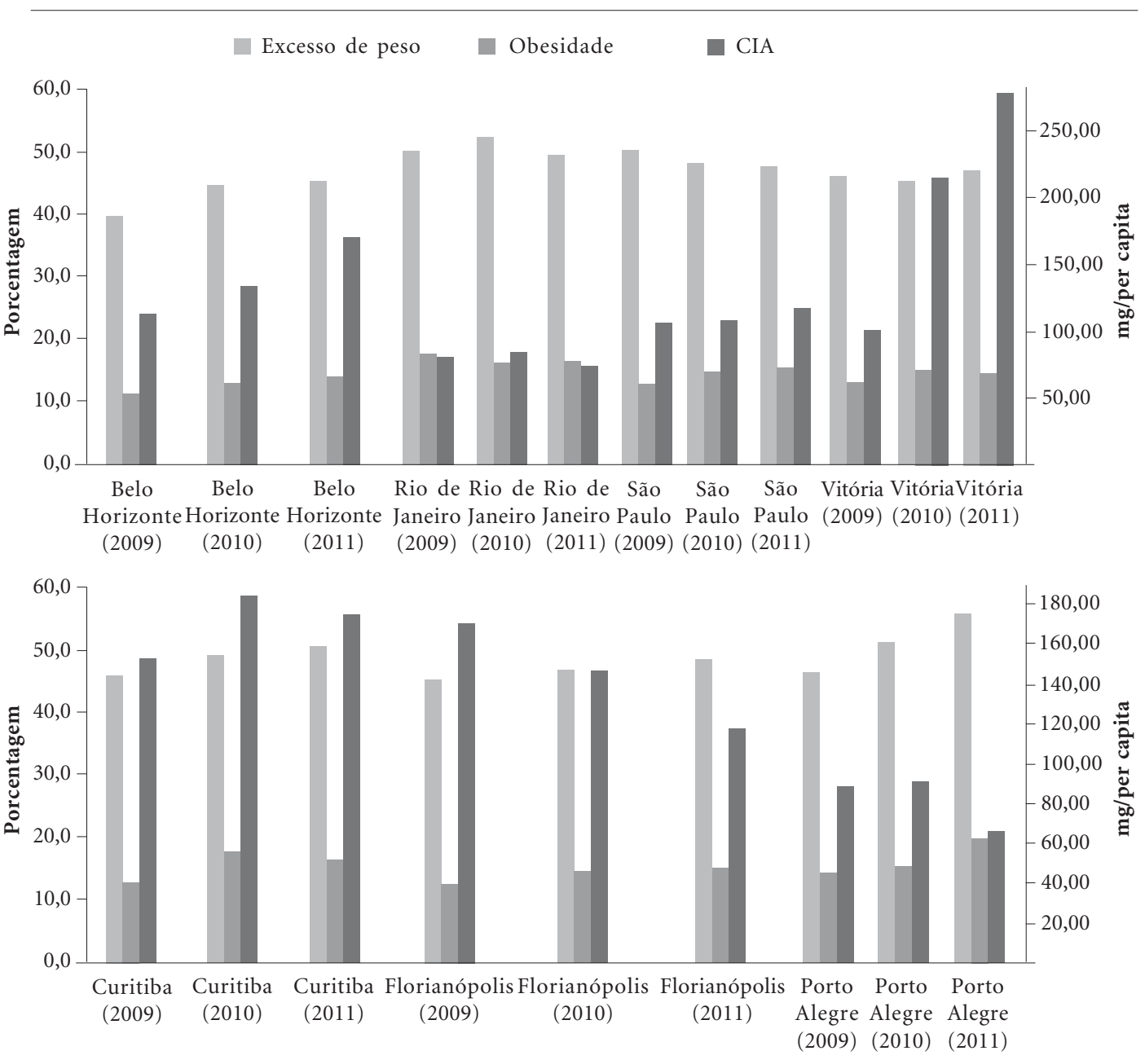

Figura 2. Distribuição de indivíduos com excesso de peso e com obesidade e do consumo de inibidores de apetite (CIA) em mg/ per capita nas capitais das regiões Sudeste e Sul, 2009-2011. 
(2010 e 2011) registraram os maiores consumos em períodos diferentes.

Na região Norte, Rio Branco (2009 e 2010) e Manaus (2011) registraram os maiores percentuais de indivíduos com excesso de peso, enquanto que para obesidade verificou-se que não houve uma predominância de nenhuma capital nos anos estudados. Porto Velho (2009), Manaus (2010) e Macapá (2011) foram as capitais com maiores registros de indivíduos obesos. A capital Palmas para ambas as condições fisiológicas estudadas obteve os menores valores. Em relação aos inibidores de apetite, Palmas teve o maior registro de consumo enquanto que Macapá (2009) e Rio Branco (2010 e 2011) registraram os menores valores.

Os dados estudados para a região Nordeste apontam que Aracaju (2009) e Fortaleza (2010 e 2011) registraram os maiores percentuais de indivíduos com excesso de peso enquanto que os menores valores foram verificados em Teresina (2009), Salvador (2010) e São Luís (2011). Em relação ao percentual de obesos, os maiores valores foram registrados em Aracaju (2009), Fortaleza (2010) e Natal (2011) e os menores percentuais foram observados em São Luís (2009), Teresina (2009 e 2011) e Salvador (2010). Quanto ao consumo de inibidores de apetite, a capital cearense registrou o menor valor em $\mathrm{mg} /$ per capita e Natal (2009 e 2011) e Teresina (2010) alcançaram a primeira colocação em anos diferentes.

A primeira estimação do modelo de regressão linear dinâmico com dados em painel demonstrou que os coeficientes das variáveis "população feminina" (valor-p =0,1427) e "excesso de peso" (valor-p $=0,6847$ ) não se mostraram estatistica- mente significativos. Uma nova estimação foi realizada sem essas variáveis, cujo processo de exclusão, uma-a-uma, obedeceu ao critério de significância estatística. Essa versão do modelo promoveu um melhor ajustamento aos dados e verificou-se, também, que não houve alteração nas variáveis que apresentaram significância estatística quando comparado ao modelo anterior (Tabela 2). O teste de Sargan (valor-p $=0,35$ ) indica que as condições de momento são válidas e, portanto, as variáveis instrumentais utilizadas na estimação são ortogonais aos resíduos da regressão.

A partir dessa estimação final do modelo, constataram-se algumas evidências sugestivas sobre o consumo de inibidores de apetite no conjunto das capitais brasileiras e do DF. Observouse que os coeficientes das variáveis assistenciais consumo de fluoxetina (lnCFF), consumo de clordiazepóxido (lnCCP) e taxa de cobertura de planos de saúde (lnPLS) apresentaram sinais positivos e foram significativos em termos estatísticos, sugerindo que o consumo de inibidores de apetite cresce com o aumento nos valores dessas variáveis. Por exemplo, o coeficiente para consumo de fluoxetina (lnCFF) foi de 0,20, indicando que a variação positiva no percentual de "consumo de fluoxetina", de 1\%, provoca um aumento médio de $0,20 \%$ no consumo de inibidores do apetite no conjunto das unidades analisadas. No caso da lnPLS, uma variação positiva na taxa percentual de cobertura de planos de saúde, de 1\%, produz uma elevação média de $0,81 \%$ no consumo de inibidores de apetite (Tabela 2).

A variável defasada $\operatorname{lnCIA}(-1)$ demonstrou que aproximadamente $79 \%$ do consumo de inibidores de apetite é recorrente, sendo fortemente depen-

Tabela 2. Estimação dinâmica final de dados em painel para as capitais brasileiras e para o DF, 2009 a 2011. ( No de observações $=81$ ). Variável dependente: Consumo de inibidores de apetite (CIA).

\begin{tabular}{lccc}
\hline \multicolumn{1}{c}{ Variável } & Coeficiente & Erro padrão & p-valor \\
\hline lnCIA $(-1)$ & 0,789562 & 0,117883 & $<0,0001^{* * *}$ \\
Constante & 0,767681 & 0,271129 & $0,0046^{* * *}$ \\
lnCFF & 0,201376 & 0,0811667 & $0,0131^{* *}$ \\
lnCCP & 0,045067 & 0,0220925 & $0,0414^{* *}$ \\
lnOBE & $-8,28636$ & 2,21194 & $0,0002^{* * *}$ \\
lnPFH & $-4,31209$ & 1,90363 & $0,0235^{* *}$ \\
lnPLS & 0,814532 & 0,395439 & $0,0394^{* *}$ \\
Teste Wald & $\chi^{2}(6)=363,098(0,0000)$ & & \\
Teste de Sargan & $\chi^{2}(1)=0,873607(0,3500)$ & & \\
\hline
\end{tabular}

${ }^{* *}$ nível significativo a $5 \%$; ${ }^{* *}$ nível significativo a $1 \%$. lnCIA $(-1)=$ logaritmo do consumo defasado de inibidores de apetite; $\operatorname{lnCFF}=$ logaritmo do consumo de fluoxetina; $\operatorname{lnCCP}=$ logaritmo do consumo de clordiazepóxido; lnOBE = logaritmo do percentual de adultos com obesidade; $\ln \mathrm{PFH}=$ logaritmo do percentual de adultos que consomem frutas e hortaliças; lnPLS = logaritmo da taxa, em percentual, de cobertura de planos de saúde. 
dente da utilização anterior desses medicamentos. Outro achado foi que a relação negativa apresentada entre as variáveis, hábitos de vida mais saudável (lnPFH) e consumo de inibidores de apetite (lnCIA), indica que, havendo um aumento médio de $1 \%$ no percentual de pessoas que consumem frutas e hortaliças, ocorre um decréscimo médio de $4,3 \%$ no consumo de inibidores de apetite no conjunto das unidades analisadas (Tabela 2).

Com relação à variável "percentual de adultos com obesidade" (lnOBE), apesar de significativa estatisticamente, apresentou outra direção do que aquela esperada. O sinal negativo do coeficiente indica que, para o cenário estudado quando ocorre um aumento médio de $1 \%$ na população de adultos obesos, há uma redução média de $8,2 \%$ no consumo de inibidores de apetite (Tabela 2).

\section{Discussão}

A tendência preocupante do aumento de pessoas com excesso de peso e obesidade tem estado na agenda de prioridades do governo brasileiro devido ao grande impacto relacionado com morbidade e mortalidade no país ${ }^{25-27}$. Os resultados deste estudo sugerem que as irracionalidades no consumo de inibidores de apetite nas capitais brasileiras e no DF podem contribuir para o agravamento desse cenário epidemiológico. As irracionalidades envolvendo hábitos de prescrição não condizente com a definição de uso racional de medicamentos proposta pela $\mathrm{OMS}^{15}$ foram observadas no consumo desses medicamentos com outros fármacos - infringindo normas legais estabelecidas pelo Conselho Federal de Medicina ${ }^{28}$ e pela Anvisa ${ }^{29}$ - e, também, na indicação terapêutica de uso tanto no que se refere aos indivíduos em tratamento como no uso continuado desses produtos.

Alguns estudos brasileiros têm sugerido a ocorrência do consumo combinado de inibidores de apetite com fluoxetina ou clordiazepóxi$\mathrm{do}^{4,6,7,9}$. Para Carlini et al. ${ }^{9}$, o medicamento fluoxetina é utilizado como sugestivo para perda de peso e não para o tratamento de depressão. Essa combinação estaria sendo utilizada como forma de obter uma maior efetividade no tratamento da obesidade ou excesso de peso em um menor espaço de tempo. Outro estudo demonstrou uma correlação positiva entre o consumo de inibidores de apetite e fluoxetina no país ${ }^{7}$. Neste estudo também foi verificada a existência de uma força de associação linear entre o consumo de inibidores de apetite e fluoxetina e, também, entre esses supressores de apetite e clordiazepóxido, sendo que esta correlação positiva foi maior com fluoxetina. As análises descritivas também contribuem para esses achados.

Um estudo realizado com 664 estudantes de uma universidade pública do estado do Piauí demonstrou que as médias de índice de massa corpórea (IMC) e circunferência da cintura (CC) foram maiores entre estudantes que referiram uso de inibidores de apetite, mas $47 \%$ deles foram classificados como eutróficos pelo IMC e 76,5\% apresentavam medida de CC normal ${ }^{3}$. Segundo ainda o estudo, com base na avaliação antropométrica daqueles que referiram indicação médica, o uso justificado de medicamentos para tratamento da obesidade ocorreu em $66,67 \%$ dos casos $^{3}$. Outro estudo apontou que $41 \%$ dos pacientes que tomavam medicamentos para obesidade não cumpriam critérios médicos necessários para sua utilização ${ }^{11}$. No presente estudo foi observado um descompasso entre o consumo de inibidores de apetite e a frequência de pessoas com excesso de peso e obesas em várias capitais brasileiras. Verificou-se também uma forte correlação inversa entre o consumo de inibidores de apetite e pacientes referidos como obesos. Esse quadro sugere que os inibidores de apetite têm sido utilizados por pessoas que não necessitam desses medicamentos para tratamento de sua queixa clínica.

Outro problema identificado foi que o consumo recente de inibidores de apetite sofreu influência do consumo passado, ou seja, o que é sugestivo de um uso continuado desses medicamentos. A segurança no uso desses medicamentos por longo prazo carece ainda de melhores evidências científicas, o que implica na necessidade de cuidados rigorosos e contínuos a serem feitos por médicos e farmacêuticos, sendo recomendado a não ultrapassar o período de tempo de tratamento estabelecido pela bula do produto. De 1995 a meados de 1997, o medicamento fenfluramina ou dexfenfluramina foi amplamente utilizado em combinação com fentermina ("fenphen"), às vezes por períodos mais longos do que algumas semanas para o tratamento da obesidade. Depois de inúmeros relatos de valvulopatia cardíaca em pessoas que fazia uso de fenfluramina ou dexfenfluramina, a Food Drug Administration (FDA) dos Estados Unidos emitiu um alerta de saúde pública, em julho de 1997, que levou à retirada voluntária dos medicamentos do mercado americano em 15 de setembro de $1997^{30}$.

Khan et al..$^{30}$, em estudo realizado nos Estados Unidos para estimar a prevalência de uso de 
medicamentos prescritos para perda de peso, verificaram um uso mais comum destes produtos entre as mulheres do que em homens. No Brasil, Carneiro et al. ${ }^{4}$ evidenciaram, em um estudo realizado em Belo Horizonte, uma predominância absoluta no consumo de inibidores de apetite pelas mulheres em relação aos homens, na razão de 10:1. A relação entre o sexo feminino e o consumo de inibidores de apetite não se mostrou estatisticamente significativa nas nossas análises. Resultado semelhante foi encontrado por Mota e Silva-Júnior ${ }^{7}$ para o país.

A correlação positiva verificada entre a taxa percentual de cobertura de planos de saúde e o consumo de inibidores de apetite condiz com as regras de acesso a esses produtos que estão preconizadas pela Portaria SVS/MS no 344/1998 ${ }^{21}$. Para o consumo desses medicamentos faz-se necessária a retenção da receita no estabelecimento farmacêutico. Os pacientes cobertos por planos de saúde nas capitais brasileiras e no DF têm melhor acesso a médicos e, consequentemente, maior facilidade na aquisição de tais medicamentos quando comparado com os de outros locais do país. No entanto, estudo publicado por Martins et al. ${ }^{3}$ mencionou uma elevada proporção de uso de inibidores de apetite sem indicação ou prescrição médica.

O comportamento de pessoas com hábitos de vida saudável demonstrou um efeito esperado com relação ao consumo de inibidores de apetite. $\mathrm{O}$ resultado sugere que os indivíduos que consomem mais frutas e hortaliças teriam menor possibilidade de ser obesos e, consequentemente, recorreriam menos à medicalização da sua saúde. Uma análise econométrica com dados dos estados americanos mostrou uma relação linear e negativamente inclinada entre o percentual de adultos que consomem frutas ou hortaliças cinco ou mais vezes por dia e a taxa de obesidade, a qual foi estatisticamente significativa ${ }^{31}$. Resultado semelhante foi obtido para excesso de peso com dados canadenses ${ }^{32}$. A prevenção e o tratamento primário da obesidade estão focados na modificação de hábitos de vida, como a adoção de uma boa alimentação à base de frutas e hortaliças e a realização de exercícios físicos. Em alguns pacientes obesos e que ademais tenham outras enfermidades ou alterações endócrinas com um IMC maior a $27 \mathrm{~kg} / \mathrm{m}^{2}$ é necessário complementar o tratamento com medicamentos ${ }^{33}$.

A partir dos resultados, recomendam-se algumas sugestões, não se limitando a elas, que poderão contribuir na redução de fatores que favorecem ao uso irracional de medicamentos, a saber: i) acompanhamento, de forma proativa do uso de inibidores de apetite pela vigilância póscomercialização do país; ii) fortalecimento da educação permanente sobre boas práticas de prescrição baseadas em evidências farmacoterapêuticas junto aos alunos e profissionais de medicina; iii) produção de informações de apoio à prescrição e utilização dos medicamentos; e, iv) estímulos a políticas sociais que contribuam com o desenvolvimento, o fortalecimento e a sustentabilidade de alternativas práticas e macroeconômicas ao uso de inibidores de apetite na redução/controle da obesidade no país, como a promoção de políticas de redução de preços de frutas e hortaliças.

Este estudo tem várias limitações que estão relacionadas com o uso de dados secundários e, em particular, envolvendo os valores do consumo dos medicamentos estudados. Primeiramente, a análise foi baseada em dados de venda que foram registrados no SNGPC, o que pode não refletir o verdadeiro consumo dos medicamentos nas capitais estudadas. Alguns problemas operacionais do SNGPC podem, ao longo dos anos, ter impedido o recebimento de arquivos contendo as movimentações de compra e venda dos medicamentos, gerando valores inferiores no consumo. Além disso, como esses produtos são objeto de interesse para o comércio clandestino, algumas unidades físicas consumidas por parte da população podem não ter sido escriturada no SNGPC, produzindo novamente um registro incompleto do consumo. No entanto, para identificar/estimar riscos para a saúde da população nunca se deve esperar em dispor de dados perfeitos, pois é pouco provável que isso ocorra ${ }^{16}$. A oportunidade na gestão de riscos sanitários em vigilância sanitária é essencial em evitar o dano ou minimizar as consequências negativas do mesmo.

Não se pode, também, excluir limitações relacionadas com as outras fontes de dados que podem ter afetados os resultados do estudo. Destaca-se ainda que as relações encontradas entre o consumo de inibidores de apetite e as variáveis independentes implicam em associações lineares e não necessariamente de causa e efeito. Fica ainda em aberto o processo de responder, em nível individual, o questionamento proposto no título do artigo, por meio de estudos farmacoepidemiológicos observacionais. Apesar disso, os estudos farmacoeconométricos podem ser úteis para identificar situações de riscos relacionados com a utilização de medicamentos, oferecendo informações adicionais sobre aspectos relativos ao consumo de medicamentos até então não observados em uma dada região. De acordo 
com Muir Gray (1997) apud Brownson et al. ${ }^{34}$, a ausência de evidência excelente não deve tornar a tomada de decisão em vigilância sanitária impossível, o que é necessário é a melhor evidência disponível que contribua com a gestão do risco, reforçando, assim, o uso saudável de medicamentos no país.

\section{Colaboradores}

DM Mota trabalhou no planejamento, na análise e na interpretação do estudo e na redação do manuscrito. MG Oliveira, RF Bovi, SF Silva e JAF Cunha participaram do planejamento e da revisão final do texto. JA Divino trabalhou na concepção do estudo, na análise e na interpretação dos dados e na revisão crítica do artigo. 


\section{Referências}

1. Junta Internacional de Fiscalização de Entorpecentes (JIFE). Relatório anual 2006. [Internet]. [acessado 2012 mar 18]. [cerca de 128 p.]. Disponível em: http://www.unodc.org/brazil/pt/pressrelease_ 20070103.html.

2. Junta Internacional de Fiscalização de Entorpecentes (JIFE). Relatório Anual 2007. O Brasil no Relatório Anual da JIFE. [Internet]. [acessado $2012 \mathrm{mar}$ 18]. [cerca de 9 p.]. Disponível em: http://www. unodc.org/pdf/brazil/JIFE/OBrasilnoRelatorio JIFE.pdf.

3. Martins MCC, Souza Filho MD, Moura FS, Carvalho JSR, Müller MC, Neves RV, Mousinho PC, Lima IP. Uso de drogas antiobesidade entre estudantes universitários. Rev Assoc Med Bras 2011; 57(5):570576.

4. Carneiro MFG, Guerra Junior AA, Acurcio FA. Prescrição, dis-pensação e regulação do consumo de psicotrópicos anorexíge-nos em Belo Horizonte, Minas Gerais, Brasil. Cad Saude Publica 2008; 24(8): 1763-1772.

5. Nappo SA, Tabach R, Noto AR, Galduróz, JCF, Carlini EA. Use of anorectic amphetamine-like drugs by Brazilian women. Eat Behav 2002; 3(2):153-165.

6. Nappo AS, Oliveira EM, Morosini S. Inappropriate prescribing of compounded antiobesity formulas in Brazil. Pharmacoepidemiol Drug Safety 1998; 7(3):207-212

7. Mota DM, Silva-Júnior GG. Evidências advindas do consumo de medicamentos moduladores do apetite no Brasil: um estudo farmacoeconométrico. Rev Assoc Med Bras 2012; 58(1):88-94.

8. Lima MS, Béria JU, Tomasi E, Mari JJ. Use of amphetamine-like appetite suppressants: a crosssectional survey in southern Brazil. Subst Use Misuse 1998; 33(8):1711-1719.

9. Carlini EA, Noto AR, Nappo AS, Sanchez ZM, Franco VLS, Silva LCF, Santos VE, Alves DC. Fluoxetina: indícios de uso inadequado. J Bras Psiquiatr 2009; 58(2):97-100.

10. Martins ELM, Amaral MPH, Ferreira MBC, Mendonça AE, Pereira MCS, Pereira DC, Ribeiro PM, Brumano LP. Dispensações de psicotrópicos anorexígenos no município de Juiz de Fora, Minas Gerais, Brasil. Cien Saude Colet 2012; 17(12):33313342.

11. Cawley J, Rizzo JA. One Pill Makes You Smaller: The Utilization of Anti-Obe-sity Drugs. Ithaca: Cornell University; 2004. (Working Paper) Dec 1-27.

12. Brasil. Agência Nacional de Vigilância Sanitária (Anvisa). Nota técnica sobre eficácia e segurança dos medicamentos inibidores de apetite. Brasília: Anvisa; 2011.

13. Kang JG, Park C. Anti-Obesity Drugs: A Review about Their Effects and Safety. Diabetes Metab J 2012; 36(1):13-25.

14. Agência Nacional de Vigilância Sanitária. Resolução RDC no 52, de 6 de outubro de 2011. Dispõe sobre a proibição do uso das substâncias anfepramona, femproporex e mazindol, seus sais e isômeros, bem como intermediários e medidas de controle da prescrição e dispensação de medicamentos que contenham a substância sibutramina, seus sais e isômeros, bem como intermediários e dá outras providências. Diário Oficial da União 2011; 10 out.
15. World Health Organization (WHO). The world medicines situation 2011 - Rational use of medicines (WHO/EMP/MIE/2011.2.2). $3^{\text {rd }}$ Edition. Geneva: WHO; 2011. [Internet]. [acessado 2013 set 12]. [22p]. Disponível em: http://apps.who.int/medicinedocs/ documents/s18064en/s18064en.pdf.

16. World Health Organization (WHO). Regional Office for South-East-Asia. National Essential Drug Policy including Rational Use of Medicines (SEA/RC64/ 16). Índia; 2011 Sep 6-9. [Internet]. [acessado 2013 fev 21] [cerca de 3 p.]. Disponível em: http://www. searo.who.int/entity/regional_committee/64/rc64_ r5.pdf.

17. Mota DM, Silva MGC, Sudo EC, Ortun V. Uso racional de medicamentos: uma abordagem econômica para tomada de decisões. Cien Saude Colet 2008; 13(Supl.):589-601.

18. Hay JW. Appropriate Econometric Methods for Pharmacoeconometric Studies of Retrospective Claims Data: An Introductory Guide [editorial]. J Manag Care Pharm 2005; 11(4):344-347.

19. Agência Nacional de Vigilância Sanitária (Anvisa). Resolução RDC nº 13, de 26 de março de 2010. Dispõe sobre a atualização do Anexo I, Listas de Substâncias Entorpecentes, Psicotrópicas, Precursoras e Outras sob Controle Especial, da Portaria SVS/MS no 344, de 12 de maio de 1998 e dá outras providências. Diário Oficial da União 2010; 30 mar.

20. Hsiao C. Analysis of Panel Data. $2^{\text {nd }}$ edition. New York: Cambridge University Press; 2003.

21. Brasil. Portaria SVS/MS $n^{\circ} 344$, de 12 de maio de 1998. Aprova o Regulamento Técnico sobre substâncias e medicamentos sujeitos a controle especial. Diário Oficial da União 1998; $31 \mathrm{dez}$.

22. Brasil. Ministério da Saúde (MS). Datasus. Informações de Saúde (TABNET). Demográficas e Socioeconômicas. [Internet]. [acessado 2014 mar 28]. Disponível em: http://www2.datasus.gov.br/DATASUS/ index.php?area $=0206$

23. Brasil. Ministério da Saúde (MS). Vigitel. [Internet]. [acessado 2014 mar 28]. Disponível em: http:/ /portal.saude.gov.br/portal/saude/Gestor/area.cfm? id_area $=1693$

24. Brasil. Ministério da Saúde (MS). Agência Nacional de Saúde Suplementar (ANS). [Internet]. [acessado 2014 mar 28]. Disponível em : http://www. ans.gov.br/anstabnet/anstabnet/deftohtm.exe? anstabnet/dados/TABNET_TX.def

25. Lessa I. Doenças crônicas não-transmissíveis no Brasil: um desafio para a complexa tarefa da vigilância. Cien Saude Colet 2004; 9(4):931-943.

26. Schmidt MI, Duncan BB, Silva GA, Menezes AM, Monteiro CA, Barreto SM, Chor D, Menezes PR. Chronic non-communicable diseases in Brazil: burden and current challenges. The Lancet 2011; 377(9781):1949-1961.

27. Sichieri R, Nascimento S, Coutinho W. The burden of hospitalization due to overweight and obesity in Brazil. Cad Saude Publica 2007; 23(7):1721-1727.

28. Conselho Federal de Medicina (CFM). Resolução CFM no. 1.477, de 11 de julho de 1997. Diário Oficial da União 1997; 16 jul. 
29. Agência Nacional de Vigilância Sanitária (Anvisa). Resolução RDC no 58, de 5 de se-tembro de 2007. Dispõe sobre o aperfeiçoamento do controle e fiscalização de substâncias psicotrópicas anorexígenas e dá outras providências. Diário Oficial da União 2007; 6 set.

30. Khan LK, Serdula MK, Bowman BA, Williamson DF. Use of prescription weight loss pills among U.S. adults in 1996-1998. Ann Intern Med 2001; 134(4):282-286.

31. Stacey J. Econometric analysis of obesity rate in the United States. $3^{\text {rd }}$ Annual Siena College Student Conference in Business. April 18, 2008. [Internet]. [acessado 2012 dez 12] [cerca de 26 p.]. Disponível em: http://www.siena.edu/uploadedFiles/Home/ Academics/Schools_and_Departments/School_of_ Business/Obesity\%20Rates\%20in\%20the\%20US\%20 by\%20Stacey.pdf.

32. Edwards M. An econometric analysis of overweight and obesity in Canada: Evidence from cycle 2.2 of the Canadian Community Health Survey. New Brunswick: University of New Brunswick. [Internet]. [acessado $2012 \mathrm{dez}$ 12]. [cerca de 69 p.]. Disponível em: http://dspace.hil.unb.ca:8080/xmlui/bitstream/ handle $/ 1882 / 43968 /$ MR56543.pdf?sequence $=$

33. Vizuet JAP. Tratamiento farmacológico del paciente con obesidad. In. González JAM, organizador. Obesidade - Un enfoque multidisciplinario. México: Universidade Autónoma del Estado de Hidalgo; 2010. p. 219-225.

34. Brownson RC, Fielding JE, Maylahn CM. EvidenceBased Public Health: A Fundamental Concept for Public Health Practice. Annu. Rev. Public Health 2009; 30:175-201.

Artigo apresentado em 17/09/2013

Aprovado em 29/11/2013

Versão final apresentada em 08/12/2013 